\title{
Autosomal dominant hypophosphatemic rickets
}

INSERM

\section{Source}

INSERM. (1999). Orphanet: an online rare disease and orphan drug data base. Autosomal dominant hypophosphatemic rickets. ORPHA:89937

Autosomal dominant hypophosphatemic rickets (ADHR) is a hereditary renal phosphatewasting disorder characterized by hypophosphatemia, rickets and/or osteomalacia. 